\title{
Immunogenetics and Infectious Diseases:Special Reference to the Mayor Histocompatibility Complex
}

\author{
Crésio Alves, Thaisa Souza, Isadora Meyer, \\ Maria Betânia P. Toralles and Carlos Brites
}

\author{
Medicine School, Federal University of Bahia; \\ Salvador, BA, Brazil
}

\begin{abstract}
Many studies have tried to identify genetic markers for infectious diseases, some of them have focused on human leukocyte antigens (HLA). The products of HLA genes interact with surfacespecific receptors of T lymphocytes, resulting in activation of the host's immune response. Association of bacterial, viral, parasitic and fungal infections with the host's HLA has been widely investigated. The type and strength of this association differs among distinct populations, as well as among racial and/or ethnic groups. The new molecular methods for the identification of the HLA alleles, and the resulting new nomenclature, have contributed to a better understanding of this system. Unfortunately, this information has not been adequately transmitted to clinicians, which hampers the understanding of the association between the HLA system and diseases. We revised relevant studies on the association of HLA genes with infectious diseases, demonstrating their importance in the pathogenic mechanisms, through increased susceptibility or protection against infections and their complications. Key Words: HLA, infectious diseases, major histocompatibility complex.
\end{abstract}

The human immune system is regulated by molecules coded by some genes, among which, are the genes of the human histocompatibility system, which code for human leukocyte antigens (HLA). These genes are located in the short arm of chromosome 6 [1] and are divided into three classes, I, II \& III [2,3]. HLA class I is responsible for coding the molecules HLA-A, -B and -C, present in almost all somatic cells $[2,3]$. The class II genes code the molecules HLA-DR, DQ and -DP, expressed in a subgroup of antigen-presenting immune cells, including B cells, activated T cells, macrophages, dendritic cells and thymic epithelial cells $[2,3]$.

HLA is the most polymorphic genetic system in the human being, with numerous alleles, and consequently, various possible combinations [1,2]. This polymorphism contributes to the genetic diversity of the species and to the differences in susceptibility to diseases among genetically distinct groups, thus making it difficult for large-scale epidemics to occur [4]. The molecules coded for by the HLA system are responsible for the presentation of antigens. The T lymphocytes only recognize antigens when they are linked to HLA molecules $[1,2]$. Due to their role in immune response activation, the HLA antigens apparently participate in controlling resistance and susceptibility to diseases [5].

There are several hypotheses about the HLA/disease association mechanism, and it is possible that this mechanism varies for different diseases. One of the hypotheses attributes a greater or less affinity of HLA for the disease-causing peptide [6]. Thus, the HLA antigens function as receptors for some etiological agents, by facilitating their entry into the cell or by

Received on 18 December 2005; revised 27 March 2006.

Address for correspondence: Dr. Crésio Alves. Rua Plínio Moscoso, 222, Apto. 601, Zip code: 40157-190. Salvador - Bahia - Brazil. Email: cresio.alves@uol.com.br

The Brazilian Journal of Infectious Diseases 2006;10(2):122-131. (C) 2006 by The Brazilian Journal of Infectious Diseases and Contexto Publishing. All rights reserved. making such entry difficult. Another possibility would be the early intervention of HLA in the thymic selection of lymphocytes, by determining which antigens will be presented to the T lymphocytes [6]. There is also the hypothesis that there may be a mechanism of tolerance of $\mathrm{T}$ cells to these pathogens, through molecular mimicking between antigens of the infectious microorganisms and antigens of the host, thus providing susceptibility or protection against these diseases [1]. We reviewed the main associations of the HLA system with infectious diseases in humans.

\section{Methods for Detecting Antigens and Alleles}

To detect HLA antigens and alleles, the following methods are available: Cellular (Terasaki microlymphocytotoxicity and lymphocyte mixed culture) and Molecular Biology (SSP: sequence-specific primers and SSOP: sequence-specific oligonucleotides probes) [6]. The cellular microlymphocytotoxicity mediated by antibody method functions through the reaction of an antibody against the HLA molecules present on the cell surface [7]. In the mixed culture of lymphocytes, the HLA specificities are defined by cells with a known phenotype. T lymphocytes are used for typing HLA-A, B and C antigens, and B lymphocytes are used for HLA-DR and -DQ antigens. There are no specific antiserums to typify HLA-DP antigens by this method [2]. The molecular biology techniques do not typify the HLA antigens expressed on the cell surfaces, but the alleles themselves or groups of alleles. After being extracted from the cells, the DNA is amplified by the polymerase chain reaction. Thereby, the typification of the HLA alleles may be done by the SSP or the SSOP methods. The former uses oligonucleotide primers with specific sequences, capable of detecting an allele or a group of alleles. The latter uses oligonucleotide probes with specific sequences, designed for recognizing one or some of the alleles of a certain group [2]. 


\section{HLA System Nomenclature}

The HLA system nomenclature is routinely updated by an international committee that promotes periodic meetings to confer new names to recently discovered genes or to change the official nomenclature [6]. The HLA antigens, defined by serology, are designated by the prefix HLA, followed by the denomination of the gene locus (e.g., HLA-A, HLA-DR) and by the numerical identification of the antigen (e.g., HLA-A1, HLA-A2). The nomenclature of the C locus incorporates the letter "w" (e.g., HLA-Cw1, -Cw2) to differentiate it from the complement system (e.g., C1, C2) [6].

The alleles are identified by molecular biology methods. The HLA class II alleles are designated by the prefix HLA and their gene locus (e.g., HLA-DQ, HLA-DR, HLA-DP), followed by the letter " $A$ " or " $B$ " to designate the polymorphic $\alpha$ and $\beta$ chains of the HLA-DP and HLA-DQ (e.g., HLA-DQA, HLADQB) and only by the letter "B" for the HLA-DR, because this is its only polymorphic chain (e.g., HLA-DRB). As some regions have various genes for the $\alpha$ and $\beta$ chains, each locus receives a number corresponding to the antigen detected (e.g., HLA-DQB1), after which an asterisk is added to define the method as being molecular biology (e.g., HLA-DQB1*). After the asterisk, two to eight digits are added (e.g., HLADQB1*0101). The first two digits refer to the serological typification of the antigen, the third and fourth to the denominations of the specific alleles, the fifth and sixth represent synonym variations, and the seventh and eighth represent variations at introns 5' or 3' of the gene [6,8]. The HLA class I alleles follow the same rule, except for the fact that in these the letters " $A$ " or " $B$ " are not added to designate the polymorphism of the chains (e.g., HLA-A*0201). When the gene does not code for a protein, the letter " $\mathrm{N}$ " (nil) is added at the end of the allele denomination (e.g., HLAA*0104N).

\section{Association of HLA With Infectious Diseases}

Recently, there has been great interest in the genetic markers that interact with other factors to determine the host's response to an infectious disease [5]. Genetic factors are one of the main determinants of susceptibility to infectious diseases in humans [9]. The same infectious agent may be capable of causing different immune responses in different infected individuals. From a genetic point of view, the HLA system would appear to be one of the main mechanisms responsible for the varied clinical forms of some infectious diseases [10]. This happens because, when the different allelic forms of the HLA molecules participate in the immune response, they play an important role in controlling resistance or susceptibility to infectious diseases [5].

In order for an efficient immune response to occur against a specific infectious agent, the HLA molecules must link to the peptides derived from this pathogen and the repertoire of the $\mathrm{T}$ cells must include clones that can be activated by this HLA-peptide association [11]. One of the advantages of the polymorphism of the HLA region appears to be to avoid deficiencies in this process [1]. In individuals whose HLA molecules do not work properly, there may be a defective immune response to pathogenic microorganisms [1]. Susceptibility to an infectious disease may be due to imperfections in some stages of this system. A person that has a certain combination of HLA alleles that do not link in an appropriate manner to the peptide, or whose HLA-peptide link does not elicit an adequate response from the lymphocytes, will be less apt to resist the invasion of the infectious agent than a person who does not have these deficiencies [11]. In patients in whom HLA provides protection, these genes probably select and stimulate $\mathrm{T}$ cells that multiply and eliminate the invading agent, through the production of inflammatory cytokines or by destroying the infected cells themselves [12].

The HLA alleles vary in ethnically different populations. Studies suggest that the alleles that confer resistance to certain pathogens are prevalent in areas where they cause endemic diseases. Greater resistance to infectious diseases occurs in persons that are heterozygote for specific HLA alleles, because a heterozygous person would have a broader spectrum of peptides to link to the T lymphocytes $[11,13]$. These alleles also vary from one disease to another, due to the differences in their pathogenesis [5]. Genomic analysis of families with multiple cases of a certain type of infection has been the method most recently used for mapping and identifying the loci that affect the susceptibility to these diseases [9]. Genetic studies of infectious diseases not only help us to gain a better understanding of the pathogenic mechanisms of diseases, they may also help with the development of vaccines.

\section{Bacterial Infections}

Tuberculosis. Tuberculosis is the result of infection by Mycobacterium tuberculosis. It may cause disease in various organs, the most common form being pulmonary. Variations in susceptibility of the organs appear to have a genetic basis related to the polymorphism of the HLA region, which suggests the participation of genetic factors influencing the host's response to infection by M. tuberculosis [1,5]. The HLA-DR2 antigen (expressed by the alleles HLA-DRB2*1501 and DRB $1 * 1502$ ) is associated with the development of severe and multibacillary forms of tuberculosis, as well as with the greater prevalence of forms resistant to drug therapy [14]. The HLA-DRB $1 * 07$ and -DQA $1 * 0101$ alleles are associated with greater susceptibility to the development of pulmonary tuberculosis and the HLA-DQA $1 * 0301$ and -DQA $1 * 0501$ alleles are associated with protection against this infection [15]. Selvaraj et al. (1996) suggested that the heterozygote combination of various HLA-DR antigens influences the 
tuberculin reaction in pulmonary tuberculosis [16]. Other alleles are not associated with the severity of tuberculosis, but they are associated with predisposition to or protection against its occurrence. Ravikumar (1999) observed associations of the HLA-DPB $1 * 04$ allele with protection against tuberculosis, while -DRB1*1501 and -DQB1*0601 are associated with susceptibility [17]. Mehra et al. (1995) reported association of the HLA-DRB1*1501 allele with susceptibility to tuberculosis, emphasizing the importance of the HLADRB1*1501-DRB5*0101-DQA1*0103-DQB1*0601 haplotype [18]. The diversity of the HLA antigens in populations from different ethnic-racial origins can be demonstrated by means of a few examples. In Poles, it was suggested that the HLADR16 antigen increases the risk of developing tuberculosis, while the antigen HLA-DR13 protects against the disease [19]. In India, the results are controversial. One study showed that the HLA-A10, -B8 and -DR2 antigens presented with greater frequency in persons with tuberculosis than in healthy controls [20], while another did not find any association between HLA class II antigens and pulmonary tuberculosis [21].

Hansen's disease. Hansen's disease (leprosy) is a chronic infectious disease caused by Mycobacterium leprae. It runs its course with lesions in various organs, depending on how the immune system responds to the presence of the mycobacteria. In infections by Mycobacterium leprae, the HLA alleles modulate the immune response, exercising an influence not only on the resistance or susceptibility to the disease, but also on the form in which it presents [10]. Some studies suggest associations of the HLA-DR2 and -DR3 antigens with the tuberculoid form and the HLA-DQ1 antigens with the virchowian form of Hansen's disease [10]. There are several studies on susceptibility to or protection against leprosy. Shankarkumar (2004) found that contaminated individuals had a greater frequency of the alleles HLA-A*0203, -A*0206, -A*1102, -B*1801, -B*4016, -B*5110, -Cw*0407 and $-\mathrm{CW} * 0703$ and a lower frequency of HLA-A*0101, -A*0211, $\mathrm{B} * 4006,-\mathrm{Cw}^{*} 03031,-\mathrm{Cw} * 04011$ and $-\mathrm{CW}^{*} 0602$ [22]. Mehra et al. (1995), in India, suggested that the HLA-DRB $1 * 1502$ allele is positively associated with the development of Hansen's disease, and that the HLA-DRB $1 * 1501-D R B 5 * 0101-$ DQA1*0102-DQB1*0502 haplotype is associated with protection [23]. In Mexico, the HLA-DRB1*1501-DQA1*0102DQB1*0602 haplotype was associated with susceptibility to leprosy [24]. In an investigation of HLA alleles and the severity of leprosy, Rani et al. (1993), in India, found a high frequency of the HLA-DRB $1 * 1501$ and -DRB $1 * 1502$ alleles in patients with the multibacillary and tuberculoid form and of DQA $1 * 0103$ in those who had the virchowian form [25]. The HLA-DQA $1 * 0102$ allele was more frequent in patients with the light form of the disease [25]. On the other hand, the HLADRB1*0701, -DQB1*0201 and-DQB1*0503 alleles were less frequent in patients with the multibacillary form, when compared with patients with the tuberculoid form; in this latter group there was a low frequency of the HLA-DQB1*0503 allele [25]. Thus, patients that presented with HLA-DR2 andDQ1 antigens, especially the HLA-DRB1*1501, -DQB1*0601 and -DQA $1 * 0103$ alleles, are considered to be more susceptible to developing severe forms of this disease, multibacillary lepromatous leprosy [25]. The HLA-DRB1*0701, -DQB1*0201 and-DQA1*0201 alleles would appear to protect against the severe forms of the disease and -DQB1*0503 against the lighter forms [25]. The HLA-A*1102-B*4006Cw*1502, HLA-A*0203-B*4016-Cw*0703 and HLA-A*11$\mathrm{B} * 40$ haplotypes were associated with protection against the multibacillary form [22]. In Brazil, Marcos et al. (2000) found no association of the ulcerated type 1 hansen reaction (manifestation of hypersensitivity due to bacillary multiplication) with HLA class II antigens [10]. However, Miller et al. (2004) suggested an association of molecules coded by genes from the HLA-DQA region with the occurrence of this disease [26]. Another study, also conducted on Brazilians, found a positive association between the HLA-DR2 antigen and the tuberculoid form of Hansen's disease [27].

Other bacteria. Bacterial infections are related to the histocompatibility antigens in several ways. The association of bacterial infections with ankylosing spondylitis, anterior uveitis and Reiter syndrome is common [28]. The Klebsiella antigens are similar to the HLA-B27 antigen, and through a cellular mimicking mechanism they contribute to the pathogenesis of ankylosing spondylitis, and they influence the frequency and age at which it develops, being earlier in HLA-B27 positive individuals [29,30]. Furthermore, the time that elapses between the appearance of the first symptoms and the diagnosis of the disease would appear to be longer in HLA-B27 negative patients. The identification of bacterial peptides that mimic HLA-B27 antigens suggests the possibility of antigens foreign to the body being a factor that sets off other spondylarthropathies [29]. In contrast, the frequency of ankylosing spondylitis development in adolescents appears to be independent of the presence of HLA-B27. In patients with anterior uveitis, there are reports of an association of infection by Gram-negative bacteria and by Bartonella henselae with the presence of the HLA-B27 antigen [28,31,32]. Anterior uveitis associated with HLAB27 is characterized by acute and recurrent episodes of pain, redness and an accumulation of cells and fibrin in the anterior chamber of the eye [33]. Although it is associated with the development of anterior uveitis, this antigen is not related to the appearance of its complications, such as posterior uveitis [34]. In 2004, Heiligenhaus et al. found an association of anterior uveitis with HLA-DR antigens, but only in patients negative for HLA-B27 [33]. Among the ones positive for HLA-DR antigens and negative for -B27, the plasmatic levels of HLA-DR antigens were much higher in those in the chronic course of the disease than in patients in the acute course. 
The Reiter syndrome is characterized by non-gonococcic urethritis, conjunctivitis and arthritis. It manifests itself from one to four weeks after an episode of genitourinary or gastrointestinal tract infection by Chlamydia, Ureoplasma, Shigella, Yersinia, among other bacteria, and its occurrence is also related to the HLA-B27 antigen. In addition, some studies suggest an association of this syndrome with the HLA-B51 antigen in Japanese patients who do not have HLA-B27 [35,36]. As they are related to the same HLA antigen, an association between ankylosing spondylitis, anterior uveitis and Reiter syndrome is common [28]. Thus, anterior uveitis frequently appears as the first indication of the concomitant presence of extra-ocular diseases, ankylosing spondylitis being the most common of them. Queiro et al. (2002) considered the HLA-DR13 antigen and extensive axial involvement in joint diseases to be the best predictors of anterior uveitis [39].

\section{Parasitic Infections}

Malaria. Malaria is an infectious disease caused by intracellular protozoans of the genus Plasmodium. Genes located in the HLA complex appear to protect populations in endemic areas against the severe forms caused by Plasmodium falciparum. In Thailand, an increased frequency of the HLA-B46 and -B56 antigens and of the HLA-DRB1*1001 allele were found among groups of patients with severe non-cerebral and cerebral malaria [40]. An association with the HLA-B53 antigen and protection against severe forms of the disease is well established, reducing the risk of death by up to $40 \%[5,11]$. The HLA-DRB1*1302-DQB1*0501 haplotype appears to protect against severe forms [11]. As this association is related to parasitic antigens present in the hepatic stage of the illness, it may influence the development of vaccines that use this antigen as one of their components [44]. In India, increased frequency of the HLA-A3, -B27 and -B49 antigens and of the HLA-DRB $1 * 04$ and -DRB $1 * 0809$ alleles were found [45]. In the same population the HLA-A19, -A34, -B18, and -B37 antigens and the HLA-DQB $1 * 0203$ allele were associated with protection [42]. Johnson et al. (2000) suggested that immunity against Plasmodium falciparum varies in accordance with the individual's age [43]. They indicated that the HLADQB1*0301 and -DQB1*03032 alleles only showed agedependent association with the levels of antibody against rRAP1 proteins of the plasmodium in children from 5 to 15 years old. After this age, immunity against malaria becomes independent of these two alleles. The HLA-DRB $1 * 03011$ allele is associated with higher levels of antibody against the parasite's rRAP2 protein in adults over 30 years of age. These data suggest that the effect of HLA on the evolution of infection by malaria differs in accordance with age, that it is stimulated by different antigens of the plasmodium, and that HLA is able to influence the quantity of antibodies to be produced against the parasite [43].
Cutaneous and Visceral Leishmaniasis. Various species of parasites of the genus Leishmania (e.g., L. brasiliensis, L. chagasi, L. donovani, L. major, L. infantum and L. mexicana) can infect humans. The clinical manifestations are very diverse, and these infections are able to cause an asymptomatic clinical condition, cutaneous and mucocutaneous infection, or visceral disease, depending on the species of parasite and on the host's immune response $[44,45]$. This heterogeneity is a reflection of the complex parasite-host interaction, influenced by the host's immune response and by the genetic factors of both species [44]. This being so, susceptibility to infection by Leishmania may be associated with some of the genes and haplotypes of the HLA regions. This is reinforced by family group studies, a high relative risk for monozygotic twins and by segregation analyses $[44,46]$. One of the first studies about the HLA-cutaneous leishmaniasis association showed the HLA-Cw7 antigen as a marker of susceptibility to this disease [47]. In Venezuela, the HLA-A11, -B5, -B7, -Bw2 and DQw3 antigens were found to be associated with an increased susceptibility to cutaneous leishmaniasis [48]. In Egypt, association with the HLA-A11, -B5 and -B7 antigens was suggested [49,50]. Petzl-Erler et al. (1991), in Brazil, showed that the HLA-DQw3 antigen was associated with greater susceptibility, and HLA-DR2, with greater protection [51]. In Iran, the HLA-A26 antigen is associated with the occurrence of kala-azar [52]. In Tunisia [44], in India [53] and in Brazil [46], no association was found between HLA classes I and II and kala-azar. In another Indian study, it was demonstrated that a greater expression of HLA-DR antigens is associated with the activation of $\mathrm{T}$ cells in the immune response against infestation by Leishmania donovani [54]. However, this study did not explain whether this fact was due only to the greater expression of HLA-DR antigens, or whether there were other factors involved in the process [54]. In Brazil, an increase in the expression of HLA-DR antigens was found in patients infected by Leishmania chagasi [55]. Also in Brazil, Bacellar et al. (1998) suggested that the HLA class I antigens are capable of suppressing the proliferation and secretion of cytokines by $\mathrm{T}$ lymphocytes in the immune response against Leishmania antigens [56].

Schistosomiasis. Schistosomiasis is one of the most important parasitic diseases in humans [57]. The form prevalent in Brazil is caused by Schistosoma mansoni [58]. In patients infected by Schistosoma mansoni, Salam et al. (1979), in Egypt, associated hepatosplenomegaly and its severity to the occurrence of the HLA-A1 (HLA-A*01) and -B5 (HLA-B*5106) antigens [59]. Hafez et al. (1991) observed a greater degree of hepatic fibrosis with the HLA-A2 (HLA-A*02) and -B12 ($B * 4409)$ antigens and a lower degree of hepatic fibrosis with the HLA-DR2 (HLA-DRB1*1603 or-DRB1*1605) antigen [60]. In Egypt, Assad-Khalil et al. (1993) showed an association of the HLA-B5 (-B*85106) and-DR3 (-DRB1*0306) antigens with schistosomiasis mansoni [61]. In Brazil, Cabello et al., (1991) 
did not find any association with the HLA-A or HLA-B antigens [58]. Schistosoma japonicum caused the most widely studied schistosomiasis, in terms of its association with the HLA system. Several studies have reported that the clinical manifestations of this disease are governed by HLA genes, which control the immune responsiveness to the parasite's antigens of the host organism [62]. The HLA-DRB1*1202 and -DQA1*0601 alleles are considered to be protectors against the advance of hepatic fibrosis, since these two alleles frequently co-occur, although their individual protective effects have not yet been explained [63]. In patients with an advanced degree of hepatic fibrosis, the presence of the HLADQB1*05031 allele is common, while the HLA-DQA1*0601 allele is considered to be protective [63]. The HLADRB1*1501-DRB5*0101 haplotype is associated with an advanced degree of hepatic fibrosis, and the HLA-DRB1*1101DQA $1 * 0501-\mathrm{DQB} 1 * 0301$, -DQA $1 * 0103-\mathrm{DPB} 1 * 0201$ haplotypes are associated with protection against fibrosis [64]. Recently McManaus et al. (2001) associated the HLADRB1*0901, -DRB1*1302, -DQB1*0303 and -DQB1*0609 alleles with greater susceptibility to advanced hepatic fibrosis [57]. The HLA-DRB1*1501 and-DQB $1 * 0601$ alleles appear to be protective [60]. Although many factors are involved in the inflammatory process of hepatic fibrosis, the HLA genes, mainly the HLA-DRB5*0101 allele, seem to be among the main factors that affect its prognosis $[64,65]$. The heterogeneity of the studies available till now on hepatic fibrosis does not allow consistent conclusions to be drawn [58].

Chagas' Disease. Chagas' disease is an infection caused by the protozoan Trypanosoma cruzi, which runs its course in three stages: acute; asymptomatic, or characterized by unspecific symptoms; and latent and chronic, with predominantly cardiac or digestive symptoms [66]. The fact that only part of the population inhabiting endemic areas becomes infected and only part of the infected individuals develop symptoms, suggests that genetic factors influence the development of the disease [67]. In Brazilians, the HLAA30 antigen confers increased susceptibility, while the HLADQB $1 * 06$ allele confers protection, irrespective of whether its form of presentation is cardiac or digestive [66]. No significant differences were found in Brazilians in the frequency of the HLA-DRB1, -DQA1 and-DQB1 alleles among patients with the asymptomatic form of the disease compared to those who developed cardiomyopathy [68]. In Mexico, a greater frequency of HLA-B39 and -DR4 antigens was found in patients with Chagas' disease [67]. The patients with cardiomyopathy exhibited a greater frequency of HLA-B35 antigens [67]. In these same patients, there was an increased frequency of the HLA-DR16 antigen when compared with asymptomatic patients and with healthy controls. Thus, the HLA-DR4 and -B39 antigens may be associated with infection by T. cruzi; -DR16 with susceptibility to the cardiac condition, and -A68 with protection against cardiomyopathy [67]. In
Venezuelans, a greater frequency of the HLA-C $* 03$ allele was observed in patients with the cardiac form of Chagas' disease [69], and a low frequency of the HLA-DRB1*14 and-DQB $1 * 0303$ alleles was found in seropositive patients, inhabitants of an endemic region, suggesting the protective effect of these alleles against chronic infection [70]. Among seropositive patients, when comparing those with and without cardiomyopathy, high frequencies of the HLA-DRB $1 * 01$,-DRB $1 * 08$ and -DQB $1 * 0501$ alleles and low frequencies of-DRB $1 * 1501$ [69] were found in the cardiomyopathic group. Another study on this population in an endemic region revealed an association of the HLADRB1*01-DQB1*0501 haplotype with cardiomyopathy [71]. The HLA-DRB1*01-DQB1*0501-DPB1*0401 haplotype is mentioned by Fernández-Mestre et al. (2002) as a marker of susceptibility to Chagas' disease in Venezuela [72], while HLA-DRB $1 * 14-D Q B 1 * 0301$ was associated with noninfection by $T$. cruzi in individuals inhabiting a highly endemic area in Peru [73]. Overtvelt et al. (2002) demonstrated that HLA class I also participates in the escape mechanism of $T$. cruzi from the immune response, associating greater expression of some of these molecules with longer persistence of the parasite in the host organism without eliciting an immune response [74]. In Chile, high levels of HLA-B40 and -Cw3 antigens were found in seropositive patients without cardiac disease, suggesting a protective function of the HLA genes responsible for the expression of these antigens [75]. When comparing chronic cardiomyopathy patients with others that presented with cardiac transplant rejection and with a third group, made up of patients with dilated cardiomyopathy secondary to another etiology, greater expression of HLA class I was perceived in the cardiomyocytes of the first two groups [76]. In contaminated but asymptomatic patients, when compared with those that developed Chagas' disease cardiomyopathy, a greater frequency of the HLA-A68 and B39 antigens was observed in the former [67]. In another study, Overtvelt et al. (1999) suggested that up-regulation of HLA-DR antigens in dendritic cells is also involved in the escape mechanism of $T$. cruzi from the host's immune response [77]. A study conducted in Brazilian patients demonstrated that those that developed the undetermined form of the disease, had low expression of the HLA-DR antigens in their monocytes [78]. The same finding was described in Mexicans with chronic Chagas' disease [79]. The diversity of these results is probably due to environmental influences, ethnic differences among the different groups, in addition to possible differences among the parasites that attack the various populations [69].

\section{Viral Infections}

Dengue. Dengue is caused by a virus that has four serotypes: 1 , 2,3 and 4 [79]. It may present as an asymptomatic infection, a self-limiting fever or as hemorrhagic fever [80]. The possibility of a related genetic factor is suggested by the association with the HLA system, found in some studies on dengue [81]. 
In Thail patients who acquired the disease after a second infection, the HLA-A*0203 allele was associated with dengue fever, irrespective of the viral serotype; $-\mathrm{A} * 0207$ with severe hemorrhagic dengue by serotypes 1 and 2; -B*51 with hemorrhagic dengue on second exposure to the virus; $-\mathrm{B} * 52$ with dengue fever in patients with secondary infection by serotypes 1 and 2, and the antigens HLA-B44, -B62, -B76 andB77 were negatively associated with the development of the disease after secondary infection [80]. In Mexico, individuals who had the HLA-DR4 antigen had 3.6 times less chance of developing hemorrhagic dengue after primary infection, when compared with individuals who did not have this antigen [82]. In a study conducted in Brazil with patients who had contracted dengue fever after the first infection with serotype 1, the HLADQ1 antigen was associated with this disease, while no significant association was found with the HLA-DR1 antigen [79].

HIV. HIV (human immunodeficiency virus) is a highly cytopathic retrovirus, with devastating effects on the immune system, making the contaminated individual vulnerable to infection by opportunistic agents. Research carried out on a simian model suggests that progression of infection by HIV takes place due to escape from the immune response mediated by the cytotoxic T lymphocytes $[83,84]$. Certain aspects of susceptibility to the disease were found to be associated with HLA molecules. If a type of HLA is very frequent in a population, there is a greater chance for a mutant virus to appear that is capable of escaping from the immune response, and there is a greater chance of its being transmitted to individuals with a similar type of HLA. It is possible for persons that have this same type of HLA to respond unsatisfactorily to infection by HIV, and those with a different HLA to be capable of a better response to viral invasion. It is also suggested that persons who are heterozygous for HLA alleles respond better to viral infection, probably because of being capable of presenting a greater number of viral antigens to the $\mathrm{T}$ lymphocytes [84]. HLA-B*35 alleles, particularly $B * 3501$, which are normally found close to $-C w * 4$ in the HLA complex, are more consistently associated with rapid progression to AIDS [85-87]. Evidence suggests that this happens by an altered linking of these histocompatibility antigens to the peptides derived from the virus, resulting in a reduced capacity by the cells to present these peptides to the T lymphocytes [86]. The HLA-B*57, $-\mathrm{B} * 14,-\mathrm{C} * 8$ alleles, and mainly $-\mathrm{B} * 27$, were found to be negatively associated with progression of the disease, and they are possible protective factors [85,87]. Apparently these genes code for HLA molecules that recognize conserved viral epitopes, as they constitute a fundamental part of the virus structure. Thus, individuals that have these genes are able to establish a more efficient immune defense against HIV [83]. Vertical transmission between mother and fetus has also been associated with HLA. The HLA-B*18 allele was associated with a significantly lower risk of early HIV-1 transmission. An increase in the early acquisition of HIV-1 was also observed in children who had the HLA-A*29 allele [88]. Transmission is increased if the fetus or the newborn has the HLADRB1*03011 allele, while the HLA-DR13 antigen and the HLADRB1*1501 allele would appear to protect against infection [89]. Some opportunistic infections that accompany AIDS have been associated with HLA. The HLA-A*3601 allele was associated with greater risk of developing tuberculosis in HIVpositive patients, while HLA-A*6802 appears to facilitate rapid progression to pleural disease and to allow mycobacterial replication that is difficult to control [90]. It was proposed that the HLA-A1, -B8, -DR3 antigens are protective factors against infection by cytomegalovirus and by the Mycobacterium avium Complex in HIV patients with severe immunodeficiency and that have not yet been submitted to highly effective antiretroviral therapy [91]. Although most studies refer to infection by HIV-1, progression of infection by HIV-2 has also been associated with HLA. In this case, the HLA-B $* 53$ allele and HLA-B*35-Cw*4 and $-\mathrm{A} * 23-\mathrm{Cw} * 7$ haplotypes were associated with a greater risk of progression to AIDS [92].

HTLV-1. The type 1 human T-cell lymphotropic virus (HTLV1) is a complex retrovirus [93] that causes manifestation of the disease in only 5\% of infected individuals [94]. It is transmitted sexually, parenterally and by breast-feeding [95]. Infection by this virus may cause myelopathy, leukemia/lymphoma of $\mathrm{T}$ cells in adults and chronic or sub-acute inflammatory syndromes (polymyositis, arthropathy, infective dermatitis and uveitis) [96]. Several studies have found association of the diseases caused by HTLV-I with genes of the HLA system. In a population sample of black Africans infected with this virus, the HLA-DR15 and -DQ1 antigens were found with greater frequency in patients with the virus when compared with healthy controls, while the HLA-DRB1*1501,-DRB1*1101 and -DQB $1 * 0602$ alleles were observed with greater frequency in patients with leukemia/lymphoma of adult T cells than in those with tropical spastic paraparesis [99]. Furthermore, the HLADRB1*1101-DQB1*0301 and -DRB1*1501-DQB1*0602 haplotypes were more frequent in patients with neoplasia than in those with myelopathy [96]. Jeffery et al. (1999), studying a Japanese population, showed that the patients with a HLA$A * 02$ allele had a $50 \%$ lower risk of developing myelopathy, while the presence of -DRB $1 * 0101$ conferred a risk two times greater, in the absence of the protective HLA-A*02 [97]. In another study, Jeffery et al. (2000) associated the HLA-Cw*08 allele with protection against the disease and low viral load in asymptomatic patients [98]. They also showed that the presence of the HLA-A*02 and $-\mathrm{CW}^{*} 08$ alleles prevented $36 \%$ of the potential cases of myelopathy and that HLA-B*5401 was associated with a greater risk of developing myelopathy [98]. In Jamaica, patients with infective dermatitis had a higher frequency of expression of HLA-DR antigens in $\mathrm{T}$ lymphocytes [99]. 
Hepatitis B. The hepatitis B virus is transmitted mainly parenterally, the sexual path being particularly relevant. In 5 to $10 \%$ of cases it may evolve to chronic liver disease [100]. Some factors apparently cause some individuals to have more propensity to developing chronic disease than others. Among these, co-infection with HIV, immunosuppression, male homosexual, advanced age at the time of infection and HLA are outstanding factors [100]. Available information suggests that individuals that are cured of hepatitis B develop a strong response by $\mathrm{T}$ cells related to specific types of class $\mathrm{I}$ and class II HLA, while patients with chronic disease develop a weak cellular response [101]. The HLA-DRB $1 * 1302[100,102]$ and $-A^{*} 0301[100]$ alleles were found to be associated with greater elimination of the viral infection. Persistence of infection was associated with HLA-B*08 and with the haplotypes HLA-A*01-B*08-DRB1*03, $-\mathrm{B} * 44-\mathrm{Cw}^{*} 1601$ and -B*44-Cw*0501 [100]. The HLA-DRB1*0301,-DQA1*0501 and -DQB1*0301 alleles are associated with susceptibility to chronic liver disease by the $B$ virus, while the HLA-DRB $1 * 1101$, -DRB1*1104 and -DQA $1 * 0301$ are associated with less evolution to chronicity [100]. In a Turkish population, the HLA-A24 and -Cw1 antigens were found to be associated with a lower risk of developing chronic hepatic disease, as were HLA-B13, -B8, -DR7, -DR13 and-DQ3 [103]. In a Korean population, the HLA-DR6 antigen, more specifically -DR13, appears to be associated with self-limiting hepatitis B[104].

Hepatitis C. Hepatitis C is the result of infection of hepatic tissue by a parenterally transmitted RNA virus. The acute stage of the infection is generally asymptomatic, although it may evolve to chronic liver disease and hepatocellular carcinoma [105]. The manner in which the disease will develop depends on the characteristics of the host, mainly its immune response. Studies conducted on human beings suggest that individuals that have a strong immune response based on cytotoxic $\mathrm{T}$ lymphocytes achieve better viral elimination. As the response of the lymphocytes depends on the HLA molecules, research has been conducted on the association of the infection with these molecules [106]. The various stages of hepatitis $C$ evolution have been found to be associated with the HLA system. The HLA-A*03, -B*27, -DRB1*0101, DRB $1 * 0401$ and -DRB1*15 alleles are associated with eliminating the virus in the acute stage of the disease [107]. The HLA-A*2301 and -Cw*04, on the contrary, are associated with the persistence of hepatitis $\mathrm{C}$ virus in the hepatic cells [106]. The HLA-DRB $1 * 13$ allele is associated with progression to chronic liver disease [108], whereas the absence of hepatic disease [105] or the light form of the disease [108] is associated with HLA-DRB1*11. Differences in the associations between the HLA alleles and hepatitis $\mathrm{C}$ were found to depend on the ethnic origin of the population. Thio et al. (2001) analyzed the differences in the frequency of the HLA alleles in populations with distinct racial and ethnic characteristics, the population sample being divided into Black and White individuals [109].
The HLA-DQB1*0301 allele was associated with more effective viral elimination in Blacks [109]. In Whites, viral suppression was associated with the HLA-DRB $1 * 0101$ allele and with the HLA-DRB $1 * 0101-D Q B 1 * 0501$ haplotype, while the permanence of the virus was associated with the HLADRB $1 * 0301$ allele and with the HLA-DRB $1 * 0301$ DQB1*0201haplotype [109]. Bosi et al. (2002) suggested that the HLA-DR13 antigen is negatively associated with the risk for vertical transmission of hepatitis $C$ virus [110]. Studies on Caucasian populations suggest an association of the HLADQB $1 * 0301$ and -DRB $1 * 11$ alleles with a self-limiting infection by the hepatitis $C$ virus [111].

\section{Conclusions}

Several infectious diseases have been associated with the HLA system. Some of them have been well investigated, others only superficially, some with strong association and others, not much. Although various factors participate in the immune response to infection (e.g., intensity of the infection and $\mathrm{T}$ cell functioning), the interaction of these mechanisms with genetic factors seems to be important in determining the evolution of the disease [5]. Furthermore, the immune response to infections varies from one individual to another, on account of the polymorphism of the genes that influence this response [98]. HLA antigens may act alone or in combination with other genes, conferring susceptibility to, or protection against, infectious diseases [67]. Knowledge of the mechanisms of genetic protection against, and susceptibility to, infectious diseases is one of the important steps towards controlling them in endemic areas, and contributes to our understanding of both the pathogenic and protective mechanisms of these processes [9]. The mechanisms of immune response to infection, as well as the means of resistance to the medications that are influenced by the HLA genes may be the key to future vaccines, using the peptides of organisms that mimic the HLA antigens [5,12].

Progress in improving molecular biology techniques and its methods of research will help explain the association of the histocompatibility system with infectious diseases. These technological advances, allied to other actions to promote health, such as basic sanitation and educating the population to adjust daily habits to combating infections, will lead to greater success, both in the treatment and the prevention of these diseases.

\section{References}

1. Furusho J.K.Y. Papel de los genes del complexo principal de histocompatibilidad en los processos infecciosos. Rev Invest Clin 2000;52(4):461-6.

2. Fernandes A., Maciel L., Foss M., Donadi E.A. Como entender a associação entre o sistema HLA e as doenças auto-imunes endócrinas. Arq Bras Endocrinol Metab 2003;47(5):60111. 
3. Klein J., Sato A. The HLA Sistem - First of two parts. N Engl J Med 2000;343(11):702-9.

4. Van Rood J.J. The impact of the HLA-system in clinical medicine. Shweiz Med Wschr 1993;123:85-92.

5. Singh N., Agrawal S., Rastogi A.K. Infectious diseases and immunity: special reference to major histocompatibility complex. Emerg Infect Dis 1997;3(1):41-9.

6. Donadi E. A. Como entender a nomenclatura e os mecanismos de associação entre os antígenos e os alelos de histocompatibilidade com as doenças. Medicina (Ribeirão Preto) 2000;33:7-18.

7. Terasaki P.I., McClelland J.D. Microdroplet assay of human serum cytotoxins. Nature 1964;204:998-1000.

8. Marsh S.G.E., Ekkehard A.D., Bodmer W.F. et al. Nomenclature for factors of the HLA system. Tissue Antigens 2002;60:407-64.

9. Hill A.V. The immunogenetics of human infectious diseases. Annu Rev Immunol. 1998; 16:593-617.

10. Marcos E.V.C., Souza F.C., Ura S., Opromolla D.V.A. Estudo de associação entre antígenos HLA e reação hansênica tipo 1 ulcerada. An Bras Dermatol 2000;75(3):283-90.

11. Klein J., Sato A. The HLA System: Second of Two Parts. N Engl J Med 2000;343(11):782-6.

12. Mack D.G., Johnson J.J., Roberts F., et al. HLA-class II genes modify outcome of Toxoplasma gondii infection. Int $\mathbf{J}$ Parasitol 1999;29:1351-8.

13. Carrington M., Nelson G.W., Martin M.P., et al. HLA and HIV-1: Heterozygote advantage and $B * 35-C w * 04$ disadvantage. Science 1999;283:1748-52.

14. Rajalingam R., Mehra N.K., Jain R.C., et al. Polymerase chain reaction-based sequence-specific oligonucleotide hybridization analysis of HLA class II antigens in pulmonary tuberculosis: relevance to chemotherapy and disease severity. J Infect Dis 1996;173:6.

15. Amirzargar A.A., Yalda A., Hajabolbaghi M., et al. The association of HLA-DRB, DQA1, DQB1 alleles and haplotype frequency in Iranian patients with pulmonary tuberculosis. Int J Tuberc Lung Dis 2004;8(8):1017-21.

16. Selvaraj P., Reetha A.M., Uma H., et al. Influence of HLA-DR and -DO phenotypes on tuberculin reactive status in pulmonary tuberculosis patients. Tuber Lung Dis 1996;77(4):369-73.

17. Ravikumar M., Dheenadhayalan V., Rajaram K., et al. Associations of HLA-DRB1, DQB1 and DPB1 alleles with pulmonary tuberculosis in south India. Tuber Lung Dis 1999;79(5):309-17.

18. Mehra N.K., Rajalingam R., Mitra D.K., et al. Variants of HLA-DR2/DR51 group haplotypes and susceptibility to tuberculoid leprosy and pulmonary tuberculosis in Asian Indians. Int J Lepr Other Mycobact Dis 1995;63(2):241-8.

19. Dubaniewicz A. HLA-DR antigens in patients with pulmonary tuberculosis in northern Poland: Preliminary report. Arch Immunol Ther Exp (Warsz) 2000;48(1):47-50.

20. Brahmajothi V., Pitchappan R.M., Kakkanaiah V.N., et al. Association of pulmonary tuberculosis and HLA in South India. Tubercle 1991;72(2):123-32.

21. Sanjeevi C.B., Narayanan P.R., Prabakar R., et al. No association or linkage with HLA-DR or -DQ genes in south Indians with pulmonary tuberculosis. Tuber Lung Dis 1992;73(5):280-4.
22. Shankarkumar U. HLA associations in leprosy patients from Mumbai, India. Lepr Rev 2004;75(1):79-85.

23. Mehra N.K., Rajalingam R., Mitra D.K., et al. Variants of HLADR2/DR51 group haplotypes and susceptibility to tuberculoid leprosy and pulmonary tuberculosis in Asian Indians. Int J Lepr Other Mycobact Dis. 1995;63(2):241-8.

24. Gorodezky C., Alaez C., Munguia A., et al. Molecular mechanisms of MHC linked susceptibility in leprosy: towards the development of synthetic vaccines. Tuberculosis (Edinb) 2004;84(1-2):82-92.

25. Rani R., Ferandez-Vina M.A., Zaheer S.A., et al.. Study of HLA class II alleles by PCR oligo-typing in leprosy patients from North India. Tissue Antigens 1993;42:133-7.

26. Miller E.N., Jamieson S.E., Joberty C., et al. Genome-wide scans for leprosy and tuberculosis susceptibility genes in Brazilians. Genes Immu 2004;5(1):63-7.

27. Visentainer J.E., Tsuneto L.T., Serra M.F., et al. Association of leprosy with HLA-DR2 in a Southern Brazilian population. Braz J Med Biol Res 1997;30(1):51-9.

28. Wakefield D., Montanaro A., McCluskey P. Acute anterior uveitis and HLA-B27. Surv Ophtalmol 1991;36:223-32.

29. Scofield R.H., Kurien B., Gross T., et al.. HLA-B27 binding of peptide from its own sequence and similar peptides from bacteria: implications for spondyloarthropathies. Lancet 1995;345:1542-4.

30. Feldtkeller E., Khan M.A., van der Heijde D., et al. Age at disease onset and diagnosis delay in HLA-B27 negative versus positive patients with ankylosing spondylitis. Rheumatol Int 2003;23(2):61-6.

31. Smith J.R. HLA-B27-associated uveitis. Ophthalmol Clin North Am 2002;15(3):297-307.

32. Kerkhoff F.T., Rothova A. Bartonella henselae associated uveitis and HLA-B27. J Ophthalmol 2000;84(10):1125-9.

33. Heiligenhaus A., Rebmann V., Neubert A., et al.. Soluble HLA class I and HLA-DR plasma levels in patients with anterior uveitis. Tissue Antigens 2004;63(4):369-75.

34. Tarasova L.N., Drozdova E.A. Clinical features of HLA-B27 positive and negative uveitis. Vestn Oftalmol 2000; $116(3): 25-7$.

35. Shimamoto Y., Sugiyama H., Hirohata S. Reiter's syndrome associated with HLA-B51. Intern Med 2000;39 (2):182-4.

36. Taniguchi Y., Yorioka N., Kyuden Y., Asakimori Y. Reiter's syndrome associated with HLA-B51: a case report. J Int Med Res 2003;31(1):55-7.

37. Monnet D., Breban M., Hudry C., et al. Ophthalmic findings and frequency of extraocular manifestations in patients with HLA-B27 uveitis: a study of 175 cases. Ophthalmology 2004;111(4):802-9.

38. Fernandez-Melon J., Munoz-Fernandez S., Hidalgo V., et al. Uveitis as the initial clinical manifestation in patients with spondyloarthropathies. J Rheumatol 2004;31(3):524-7.

39. Queiro R., Torre J.C., Belzunegui J., et al. Clinical features and predictive factors in psoriatic arthritis-related uveitis. Semin Arthritis Rheum 2002;31(4):264-70.

40. Hananantachai H., Patarapotikul J., Ohashi J., et al. Polymorphisms of the HLA-B and HLA-DRB1 Genes in Thai Malaria Patients. Jpn J Infect Dis 2005;58(1):25-8.

41. Hill A.V.S., Elvin J., Willis A.C., et al. Molecular analysis of the association of HLA-B53 and resistance to severe malaria. Nature 1992;360:434-9. 
42. Shankarkumar U., Devaraj J.P., Ghosh K., et al. HLA associations in P. falciparum malaria patients from Mumbai, western India. Indian J Malariol 2002;39(3-4):76-82.

43. Johnson A., Leker R., Harun L., et al. Interaction of HLA and age on levels of antibody to Plasmodium falciparum rhoptryassociated proteins 1 and 2. Infect Immun 2000, 68(4):2231-6.

44. Meddeb-Garnaoui A., Gritli S., Garbouj S., et al. Association analysis of HLA-class II and class III gene polymorphisms in the susceptibility to Mediterranean visceral leishmaniasis. Hum Immunol 2001;62(5):509-17.

45. Olivo-Díaz A., Debaz H., Alaez C., et al.. Role of HLA class II alleles in susceptibility to and protection from localized cutaneous leishmaniasis. Hum Immunol 2004;65:255-61.

46. Peacock C.S., Sanjeevi C.B., Shaw M.A., et al. Genetic analysis of multicase families of visceral leishmaniasis in northeastern Brazil: no major role for class II or class III regions of HLA. Genes Immun 2002;3(6):350-8.

47. Barbier D., Demenais F., Lefait J.F., et al. Susceptibility to human cutaneous leishmaniasis and HLA, Gm, Km markers. Tissue Antigens 1987;30(2):63-7.

48. Lara M., Layrisse Z., Scorza J.V., et al. Immunogenetics of human American cutaneous leishmaniasis- Study of HLA haplotypes in 24 families from Venezuela. Hum Immunol 1991;30:129-35.

49. Morsy T.A., Romia S.A., Al-Ganayni G.A., et al. Histocompatibility antigens (HLA) in Egyptians with two parasitic skin diseases (scabies and leishmaniasis). J Egypt Soc Parasitol 1990;20:565-72.

50. El-Mogy M.H., Abdel-Hamid I.A., Abdel-Razie M.M., et al. Histocompatibility antigens in Egyptians with cutaneous leishmaniasis: a preliminary study. J Dermatol Sci 1993;5(2):89-91.

51. Petzl-Erler M.L., Belich M.P., Queiroz-Telles F. Association of mucosal leishmaniasis with HLA. Hum Immunol 1991;32(4):254-60.

52. Faghiri Z., Tabei S.Z., Taheri F. Study of the association of HLA class II antigens with kala azar. Hum Hered 1995;45:258-61.

53. Singh N., Sundar S., Williams F., et al. Molecular typing of HLA class I and class II antigens in Indian kala-azar patients. Trop Med Int Health 1997;2(5):468-71.

54. Lal S., Bimal S., Sinha A.N., Prasad L.S. Role of HLA-DR antigen on T-cell activation in visceral leishmaniasis. Indian J Exp Biol 1991;29(12):1101-3.

55. De Almeida M.C., Cardoso S.A., Barral-Netto M. Leishmania chagasi infection alters the expression of cell adhesion and co-stimulatory molecules on human monocyte and macrophage. Int J Parasitol 2003;33(2):153-62.

56. Bacellar O., Russo C., Carvalho E.M. Regulation of T cell response to leishmania antigens by determinants of histocompatibility leukocyte class I and II molecules. Modulation of response to leishmania antigen by HLA class I molecules. Braz J Med Biol Res 1998;31:1575-81.

57. McManus D.P., Ross A.G., Williams G.M., et al.. HLA class II antigens positively and negatively associated with hepatosplenic schistosomiasis in a Chinese population. Int J Parasitol 2001;31(7):674-80.

58. Cabello P.H., Krieger H., Lopes J.D., Sant'ana E.J. On the association between HLA-A1 and B5 and clinical forms of schistosomiasis mansoni. Mem Inst. Oswaldo Cruz 1991;86(1):37-40.
59. Salam E.A., Ishaac S., Mahmoud A.A. Histocompatibilty-linked susceptibility for hepatospleenomegaly in human schistosomiasis mansoni. J Immunol 1979;123(4):1829-31.

60. Hafez M., Aboul Hassan S., El-Tahan H., et al. Immunogenetic susceptibility for post-schistosomal hepatic fibrosis. Am J Trop Med Hyg 1991;44(4):424-33.

61. Assaad-Khalil S.H., Helmy M.A., Zaki A., et al.. Some genetic, clinical and immunologic interrelations in schistosomiasis mansoni. Ann Biol Clin (Paris) 1993;51(6):619-26.

62. Ohta N., Hayashi M., Tormis L.C., et al. Immunogenetic factors involved in the pathogenesis of distinct clinical manifestations of schistosomiasis japonica in the Philippine population. Trans R Soc Trop Med Hyg 1987;81(2):292-6.

63. Waine G.J., Ross A.G., Williams G.M., et al. HLA class II antigens are associated with resistance or susceptibility to hepatosplenic disease in a Chinese population infected with Schistosoma japonicum. Int $\mathrm{J}$ Parasitol 1998;28(4):537-42.

64. Hirayama K., Chen H., Kikuchi M., et al. HLA-DR-DQ alleles and HLA-DP alleles are independently associated with susceptibility to different stages of post-schistosomal hepatic fibrosis in the Chinese population. Tissue Antigens 1999;53(3):269-74.

65. Hirayama K. Immunogenetic analysis of post-schistosomal liver fibrosis. Parasitol Int 2004;53(2):193-6.

66. Dehaide N.H.S., Dantas R.O., Donadi E.A. HLA Class I and II Profiles of Patients Presenting with Chagas' Disease. Dig Dis Sci 1998;43(2):246-52.

67. Cruz-Robles D., Reyes P.A., Monteón-Padilla V.M., et al. MHC Class I and Class II genes in Mexican patients with Chagas' Disease. Hum Immunol 2004;65:60-5.

68. Faé K.C., Drigo S.A., Cunha-Neto E., et al. HLA and b-myosin heavy chain do not influence susceptibility to Chagas' disease cardiomyopathy. Microbes Infect 2000;2:745.51.

69. Layrisse Z., Fernandez M.T., Montagnani S., et al. HLA-C*03 Is a risk factor for cardiomyopathy in Chagas' disease. Hum Immunol 2000;61:925-9.

70. Fernandez-Mestre M.T., Layrisse Z., Montagnani S., et al. Influence of the HLA class II polymorphism in chronic Chagas' disease. Parasite Immunol 1998 Apr;20(4): 197-203.

71. Colorado I.A., Acquatella H., Catalioti F., et al. HLA class II DRB1, DQB1, DPB1 polymorphism and cardiomyopathy due to Trypanosoma cruzi chronic infection. Hum Immunol 2000;61(3):320-5.

72. Fernández-Mestre M.T., Jaraquemada D., Bruno R.E., et al. Analysis of the T-cell receptor $\mathrm{b}$-chain variable-region (V) repertoire in chronic human Chagas' disease. Tissue Antigens 2002; 60:10-5.

73. Nieto A., Beraun Y., Collado M.D., et al. HLA haplotypes are associated with differential susceptibility to Trypanosoma cruzi infection. Tissue Antigens 2000;55(3):195-8.

74. Overtvelt L.V., Andrieu M., Verhasselt V., et al. Trypanosoma cruzi down-regulates lipopolysaccharide-induced MHC class I on human dendritic cells and impairs antigen presentation to specific CD8 + T lymphocytes. Int Immunol 2002; 14(10):1135-44.

75. Llop E., Rothhammer F., Acuna M., et al. HLA antigens in Chagas cardiomyopathy: new evidence based on a casecontrol study. Rev Med Chil 1991;119(6):633-6. 
76. Benvenuti L.A., Higuchi M.L., Reis M.M. Upregulation of adhesion molecules and class I HLA in the myocardium of chronic chagasic cardiomyopathy and heart allograft rejection, but not in dilated cardiomyopathy. Cardiovasc Pathol 2000;9(2):111-7.

77. Overtvelt L.V., Vanderheyde N., Verhasselt V., et al. Trypanosoma cruzi infects human dendritic cells and prevents their maturation: inhibition of cytokines, HLADR, and co-stimulatory molecules. Infect Immun 1999;67(8):4033-40.

78. Souza P.E.A., Rocha M.O.C., Rocha-Vieira E., et al. Monocytes from patients with indeterminate and cardiac forms of Chagas' disease display distinct phenotypic and functional characteristics associated with morbidity. Infect Immun 2004;72(9):5283-91.

79. Polizel J.R., Bueno D., Visentainer J.E., et al. Association of human leukocyte antigen DQ1 and dengue fever in a White Southern Brazilian population. Mem Inst Oswaldo Cruz. 2004;99(6):559-62.

80. Stephens H.A., Klaythong R., Sirikong M., et al. HLA-A and $B$ allele associations with secondary dengue virus infections correlate with disease severity and the infecting viral serotype in ethnic Thais. Tissue Antigens 2002;60(4):309-18.

81. Loke H., Bethell D.B., Phuong C.X., et al. Strong HLA class $\mathrm{I}-$ restricted $\mathrm{T}$ cell responses in dengue hemorrhagic fever: a double-edged sword? J Infect Dis 2001;184(11):1369-73.

82. LaFleur C., Granados J., Vargas-Alarcon G., et al. HLA-DR antigen frequencies in Mexican patients with dengue virus infection: HLA-DR4 as a possible genetic resistance factor for dengue hemorrhagic fever. Hum Immunol. 2002;63(11):1039-44.

83. McMichael A., Klenerman P. HIV/AIDS. HLA leaves its footprints on HIV. Science 2002;296(5572):1410-1.

84. Carrington M., O'Brien S.J. The influence of HLA genotype on AIDS. Annu Rev Med 2003;54:535-51.

85. Gao X., Nelson G.W., Karacki P., et al. Effect of a single amino acid change in MHC class I molecules on the rate of progression to AIDS. N Engl J Med. 2001;344(22):1668-75.

86. Turner D. The human leucocyte antigen (HLA) system. Vox Sanguinis 2004, (Suppl 1):S87-S90.

87. Paranjape R.S. Immunopathogenesis of HIV infection. Indian J Med Res 2005;121(4):240-55.

88. Farquhar C., Rowland-Jones S., Mbori-Ngacha D., et al. Human leukocyte antigen (HLA) B*18 and protection against mother-to-child HIV type 1 transmission. AIDS Res Hum Retroviruses 2004;20(7):692-7.

89. Winchester R., Chen Y., Rose S., et al. Major histocompatibility complex class II DR alleles DRB $1 * 1501$ and those encoding HLA-DR 13 are preferentially associated with a diminution in maternally transmitted human immunodeficiency virus 1 infection in different ethnic groups: determination by an automated sequence-based typing method. Proc Natl Acad Sci 1995;92:12374-8.

90. Louie L.G., Hartogensis W.E., Jackman R.P., et al. Mycobacterium tuberculosis/HIV-1 coinfection and disease: role of human leukocyte antigen variation. J Infect Dis 2004;189(6):1084-90.

91. Price P., Keane N.M., Stone S.F., et al. MHC haplotypes affect the expression of opportunistic infections in HIV patients. Hum Immunol 2001;62(2):157-64.
92. Diouf K., Sarr A.D., Eisen G., et al. Associations between MHC class I and susceptibility to HIV-2 disease progression. J Hum Virol 2002;5(1):1-7.

93. Bangham C.R. HTLV-1 infections. J Clin Pathol 2000;53(8):581-6.

94. Borducchi D.M., Gerbase-DeLima M., Morgun A., et al. Human leucocyte antigen and human T-cell lymphotropic virus type 1 associated diseases in Brazil. Br J Haematol 2003;123(5):954-5.

95. Imirizaldu J.J.Z., Esteban J.C.G., Axpe I.R., et al. Posttransplantation HTLV-1 myelopathy in three recipients from a single donor. J Neurol Neurosurg Psychiatry 2003; 74:1080-4.

96. Manns A., Hanchard B., Morgan O.S., et al. Human leukocyte antigen class II alleles associated with human T-cell lymphotropic virus type I infection and adult T-cell leukemia/lymphoma in a Black population. J Natl Cancer Inst 1998;90(8):617-22.

97. Jeffery K.J., Usuku K., Hall S.E., et al. HLA alleles determine human T-lymphotropic virus-I (HTLV-I) proviral load and the risk of HTLV-I-associated myelopathy. Proc Natl Acad Sci U S A. 1999;96(7):3848-53.

98. Jeffery K.J., Siddiqui A.A., Bunce M., et al. The influence of HLA class I alleles and heterozygosity on the outcome of human $\mathrm{T}$ cell lymphotropic virus type I infection. J Immunol 2000;165(12):7278-84.

99. La Grenade L., Manns A., Fletcher V., et al. Clinical, pathologic, and immunologic features of human T-lymphotrophic virus type I-associated infective dermatitis in children. Arch Dermatol 1998; 134(4):439-44.

100. Thio C.L., Thomas D.L., Karacki P., et al. Comprehensive analysis of class I and class II HLA antigens and chronic hepatitis B virus infection. J Virol 2003;77(22):12083-7.

101. Jiang Y.G., Wang Y.M., Liu T.H., Liu J. Association between HLA class II gene and susceptibility or resistance to chronic hepatitis B. World J Gastroenterol 2003;9(10):2221-5.

102. Thursz M.R., Kwiatkowski D., Allsopp C.E., et al. Association between an MHC class II allele and clearance of hepatitis B virus in Gambia. N Engl J Med. 1995;332(16):1065-9.

103. Karan M.A., Tascioglu N.E., Ozturk A.O., et al. The role of HLA antigens in chronic hepatitis B virus infection. J Pak Med Assoc 2002;52(6):253-6.

104. Ahn S.H., Han K.H., Park J.Y., et al. Association between hepatitis B virus infection and HLA-DR type in Korea. Hepatology 2000;31(6):1371-3.

105. Yenigun A., Durupinar B. Decreased frequency of the HLADRB $1 * 11$ allele in patients with chronic hepatitis $\mathrm{C}$ virus infection. J Virol 2002;76(4):1787-9.

106. Thio C.L., Gao X., Goedert J.J., et al. HLA-Cw*04 and hepatitis C virus persistence. J Virol 2002;76(10):4792-7.

107. McKiernan S.M., Hagan R., Curry M., et al.. Distinct MHC class I and II alleles are associated with hepatitis $\mathrm{C}$ viral clearance, originating from a single source. Hepatology 2004;40(1):108-14.

108. Kryczka W., Brojer E., Kalinska A., et al. DRB1 alleles in relation to severity of liver disease in patients with chronic hepatitis C. Med Sci Monit 2001;Suppl 1:217-20.

109. Thio C.L., Thomas D.L., Goedert J.J., et al. Racial differences in HLA class II associations with hepatitis $\mathrm{C}$ virus outcomes. J Infect Dis 2001;184(1):16-21.

110. Bosi I., Ancora G., Mantovani W., et al. HLA DR13 and HCV vertical infection. Pediatr Res 2002;51(6):746-9.

111. Yee L.J. Host genetic determinants in hepatitis $C$ virus infection. Genes Immun 2004;5(4):237-45. 